\title{
Multisensor Fusion Fault-Tolerant Control with Diagnosis via a Set Separation Principle
}

\author{
José A. De Doná, Maria M. Seron, Alain Yetendje
}

\begin{abstract}
In this paper, a multisensor fusion fault-tolerant control system with fault detection and isolation via set separation is presented. The fault detection and isolation unit verifies that for each sensor-estimator combination, the estimation tracking errors lie inside pre-computed sets and discards faulty sensors when their associated estimation tracking errors leave the sets. An active fault tolerant controller is obtained, where the remaining healthy estimates are combined using a technique based on the optimal fusion criterion in the linear minimum-variance sense, recently proposed in the literature. The fused estimates are then used to implement a state feedback tracking controller. We ensure closed-loop stability and good performance under the occurrence of abrupt sensor faults.
\end{abstract}

\section{INTRODUCTION}

Multisensor strategies are widespread in the research community in order to improve systems reliability. Sensor fusion is one of the most used techniques for integrating data provided by various sensors, in order to obtain the best estimate [1]-[3]. However, less attention has been focused on the case when sensors are used in a feedback control system. As is the case for all automatic control system components, and sensors in particular, faults can deteriorate the performance and even jeopardize the stability of the whole system. Therefore, it is important to consider the fault tolerance capabilities of a control system at the design stage.

Fault tolerant control systems (FTCS) are control systems that possess the ability to automatically accommodate system component faults. They are capable of maintaining the system stability and acceptable performance in the event of faults; in some circumstances, reduced performance could be accepted as a trade-off. FTCS can be classified into passive FTCS and active FTCS [4]. In passive FTCS, the controller has a fixed structure and parameters, and is designed to be robust against a class of presumed faults. In active FTC, two stages are generally necessary: diagnosis and reconfiguration. The diagnosis stage is aimed at detecting, identifying and isolating the fault while the reconfiguration stage consists in adapting the controller to the faulty situation so that the overall system continues to satisfy its goals.

In this paper, we present an active fault tolerant control scheme consisting of multiple sensors-estimator combinations, a fault detection and isolation (FDI) unit, an estimate fusion mechanism with reconfiguration capabilities and a state feedback controller with reference tracking.

Research supported by CDSC, The University of Newcastle, and the Australian Research Council

The authors are with ARC Centre of Excellence for Complex Dynamic Systems and Control (CDSC), School of Electrical Engineering and Computer Science, The University of Newcastle, Callaghan, NSW 2308, Australia (email:alain.yetendjelemegni@ studentmail.newcastle.edu.au).
The FDI unit is based on invariant set computation (see, e.g., [5]). The unit tests that for each sensors-estimator combination, the estimation tracking errors lie inside precomputed "healthy" sets and discards faulty sensors when their associated estimation tracking errors leave the healthy sets to converge towards "under-fault" sets. The proposed technique is suitable for reference tracking problems, especially when the reference signal contains an offset component, since the latter ensures the separation between healthy and under-fault sets.

In the reconfiguration stage, the estimates deemed "healthy" by means of the FDI test are fused based on the optimal fusion steady-state Kalman filter proposed in [6]. The choice of this fusion filter rather than any other decentralised fusion method is motivated by the reduction of on-line computation constraints. Indeed, decentralised fusion timevarying Kalman filter algorithms [7], [8] yield large on-line computational burden resulting from on-line computation of Riccati equations, Kalman filter gains, and weighting matrices or covariances. In the case of the optimal fusion steady-state Kalman filter, on the other hand, gains and covariance matrices are constant and can be pre-computed off line. The optimal fusion steady-state Kalman filter is composed of two layers where the first layer has a netted parallel structure to determine the steady-state cross-covariance between any two faultless sensors. Estimates and covariances of all local subsystems, and the cross-covariances among the local subsystems from the first fusion layer are fused in the second fusion layer to determine the optimal steadystate matrix weights required to obtain the optimal fusion steady-state estimate. Since the steady-state covariances, cross-covariance matrices and weights can be computed offline for each healthy configuration considered, the on-line reconfiguration task simply consists of selecting the suitable optimal steady-state weighting matrix that corresponds to the group of healthy sensors diagnosed by the FDI unit. Finally, the optimal fused estimate is used to implement a state feedback controller that achieves reference tracking. Proofs of fault tolerance and stability of the resulting closedloop system are given under a set of conditions on the system parameters, such as disturbance bounds, reference signal offset and bounds, etc.

The remainder of this paper is organised as follows. Section II presents the proposed multisensor fusion scheme. The fault detection and isolation method is discussed in Section III, as well as an in depth analysis of the closed-loop behaviour in the fault-free case and when a sensor or group of sensors fails. Proofs of fault tolerance and closed-loop 
stability are also given in Section III. An example illustrating the multisensor fusion fault tolerant control strategy is shown in Section IV. Conclusions are given in Section V.

\section{Multisensor FUSION SCHEME}

\section{A. Problem formulation}

Consider the discrete-time linear time-invariant plant

$$
x(t+1)=A x(t)+B u(t)+E w(t)
$$

where $A, B$ and $E$ are constant matrices with compatible dimensions, $x(t) \in \mathbb{R}^{n}$ is the system state, $u(t) \in \mathbb{R}^{m}$ is the control input and $w(t) \in \mathbb{R}^{r}$ is a process disturbance, which is componentwise bounded as ${ }^{1}$

$$
|w(t)| \leq \bar{w}, \quad \text { for all } t \geq 0
$$

for some known constant nonnegative vector $\bar{w} \in \mathbb{R}^{r}$.

The control objective is to track a reference signal $x_{r e f}$ that satisfies the following dynamics:

$$
x_{r e f}(t+1)=A x_{r e f}(t)+B u_{r e f}(t)
$$

We consider that the reference signals are bounded according to the following assumption.

Assumption 2.1: The input reference $u_{r e f}(t)$ and the state reference $x_{r e f}(t)$ are bounded signals. In particular, constant vectors $x_{r e f, 0} \in \mathbb{R}^{n}$ and $\bar{x}_{r e f} \in \mathbb{R}^{n}$ are known such that, for all discrete time instants $t \geq 0, x_{\text {ref }}(t) \in X_{\text {ref }} \triangleq$ $\left\{x \in \mathbb{R}^{n}:\left|x-x_{r e f, 0}\right| \leq \bar{x}_{r e f}\right\}$.

\section{B. Sensor measurements}

We consider a bank of output equations that combine several sensor measurements as follows:

$$
y_{i}(t)=\Pi_{i}\left(C_{i} x(t)+\eta_{i}(t)\right)+\left(I_{p_{i}}-\Pi_{i}\right) \eta_{i}^{F}(t)
$$

for $i=1, \ldots, N$, where $y_{i}(t) \in \mathbb{R}^{p_{i}}$ is the measured output of the $i$ th group of sensors; $\eta_{i}, \eta_{i}^{F} \in \mathbb{R}^{p_{i}}$ are measurement disturbances satisfying the bounds

$$
\left|\eta_{i}(t)\right| \leq \bar{\eta}_{i}, \quad\left|\eta_{i}^{F}(t)\right| \leq \bar{\eta}_{i}^{F}, \quad \text { for all } t \geq 0
$$

where $\bar{\eta}_{i} \in \mathbb{R}^{p_{i}}$ and $\bar{\eta}_{i}^{F} \in \mathbb{R}^{p_{i}}$, for $i=1, \ldots, N$, are known constant nonnegative vectors; and $I_{p_{i}}$ is the $p_{i} \times p_{i}$ identity matrix. The fault matrix $\Pi_{i} \in \mathbb{R}^{p_{i} \times p_{i}}$ in (4) characterises the sensor fault situation, and is described as follows:

$$
\Pi_{i}= \begin{cases}I_{p_{i}} & \text { if all sensors are healthy } \\ \operatorname{diag}\left\{\pi_{i 1}, \ldots, \pi_{i p_{i}}\right\} & \text { otherwise }\end{cases}
$$

where $\pi_{i j} \in[0,1]$, for $j=1, \ldots, p_{i}$. Notice in (4) that $\pi_{i j}<1$ indicates that the $j$ th sensor of the $i$ th sensor group has lost effectiveness, and that $\pi_{i j}=0$ corresponds to outage of the sensor. The pairs $\left(A, C_{i}\right)$ are assumed observable.

\footnotetext{
${ }^{1}$ In the sequel, inequalities and absolute values are considered componentwise.
}

\section{Optimal fusion steady-state Kalman estimator}

In accordance with the previous measurement equations, we consider a bank of $N$ steady-state Kalman estimators, where each estimator is associated with one group of sensors and is designed in order to estimate the states of the system (1). To obtain the Kalman estimators we assume, temporarily and only for design purposes, that the process disturbance $w(t)$ in (1) is a zero-mean Gaussian white-noise with covariance $Q$, and that the measurement disturbances $\eta_{i}$ in (4) are zero-mean Gaussian white-noises with covariances $R_{i}$, for $i=1, \ldots, N$, uncorrelated from the process noise $w(t)$. (For the remainder of the paper, in connection with the FTC capabilities of the scheme, we will remove this assumption and only assume (2) and (5), without any stochastic description of the disturbances being required.)

The steady-state estimators are then described by the following equations [2], [6]:

$$
\begin{gathered}
\hat{x}_{i}(t+1 \mid t)=A \hat{x}_{i}(t \mid t)+B u(t) \\
\hat{x}_{i}(t \mid t)=\hat{x}_{i}(t \mid t-1)+M_{i}\left[y_{i}(t)-C_{i} \hat{x}_{i}(t \mid t-1)\right] \\
M_{i}=P_{i} C_{i}^{T}\left(C_{i} P_{i} C_{i}^{T}+R_{i}\right)^{-1} \\
P_{i}=A\left[P_{i}-P_{i} C_{i}^{T}\left(C_{i} P_{i} C_{i}^{T}+R_{i}\right)^{-1} C_{i} P_{i}\right] A^{T}+E Q E^{T} \\
P_{i i}=P_{i}-P_{i} C_{i}^{T}\left(C_{i} P_{i} C_{i}^{T}+R_{i}\right)^{-1} C_{i} P_{i}
\end{gathered}
$$

where $M_{i}$ is the optimal steady-state innovation gain, $P_{i}$ is the steady-state prediction error variance matrix and $P_{i i}$ is the steady-state estimation error variance matrix. The steady-state error cross-covariance between the $i$ th and $j$ th estimators satisfies [6]

$$
P_{i j}=\left[I-M_{i} C_{i}\right]\left[A P_{i j} A^{T}+E Q E^{T}\right]\left[I-M_{j} C_{j}\right]^{T}
$$

The following theorem provides the optimal information fusion criterion in the linear minimum-variance sense [2].

Theorem 2.2 (Optimal steady-state fusion estimate [2]): Let $\hat{x}_{i}(t \mid t), i=1,2, \ldots, \ell$, be unbiased estimators of an $n$-dimensional vector $x(t)$. Let the estimation errors be

$$
\tilde{x}_{i}(t \mid t) \triangleq x(t)-\hat{x}_{i}(t \mid t)
$$

Assume that $\tilde{x}_{i}(t \mid t)$ and $\tilde{x}_{j}(t \mid t),(i \neq j)$ are correlated, and let the covariance $\left(P_{i i}\right)$ and cross-covariance matrices $\left(P_{i j}\right)$ be given by (11) and (12), respectively. Then the optimal linear minimum-variance information fusion estimator is given by

$$
\hat{x}_{f u s}(t)=\lambda_{1} \hat{x}_{1}(t \mid t)+\lambda_{2} \hat{x}_{2}(t \mid t)+\ldots+\lambda_{\ell} \hat{x}_{\ell}(t \mid t)
$$

where the optimal matrix weights $\lambda_{i}, i=1,2, \ldots, \ell$, are computed from

$$
\lambda=\Sigma^{-1} e\left(e^{T} \Sigma^{-1} e\right)^{-1}
$$

where $\lambda=\left[\begin{array}{lll}\lambda_{1}^{T} & \ldots & \lambda_{\ell}^{T}\end{array}\right]^{T}$ and $e=\left[\begin{array}{lll}I_{n} & \ldots & I_{n}\end{array}\right]^{T}$ are both $n \ell \times n$ matrices, and $\Sigma=\left(P_{i j}\right)_{i, j=1,2, \ldots, \ell}$, is an $n \ell \times n \ell$ matrix. The corresponding estimation error covariance of the optimal fusion estimator is

$$
P_{f u s}=\left(e^{T} \Sigma^{-1} e\right)^{-1}
$$


and satisfies $P_{\text {fus }} \leq P_{i i}, i=1,2, \ldots, \ell$.

Every estimator (7)-(10) independently estimates the states of system (1) and gives the unbiased state estimate $\hat{x}_{i}(t \mid t)$ to be used in the fusion estimate (14). Only "healthy" estimates, as diagnosed by a fault detection and isolation mechanism (described in Section III below), are fused. That is, for the configuration of groups of sensors deemed healthy by the FDI, the appropriate cross-covariance constant matrix is selected in the first fusion layer and the suitable optimal steady-state matrix weights are chosen in the second fusion layer in order to obtain the optimal fusion estimate.

Thus, the fusion estimate (14) is computed over only healthy groups of sensors, that is, groups whose indices belong to the set

$$
\mathbb{H} \triangleq\{k \in\{1,2, \ldots, N\} \text { : sensor group } k \text { is healthy }\}
$$

yielding

$$
\hat{x}_{f u s}(t)=\sum_{k \in \mathbb{H}} \lambda_{k} \hat{x}_{k}(t \mid t)
$$

Note from (15) (with the index set $\{1, \ldots, \ell\}$ redefined to be $\mathbb{H})$ that

$$
e^{T} \lambda=\sum_{k \in \mathbb{H}} \lambda_{k}=I_{n}
$$

\section{Prediction and estimation errors}

We now define the prediction errors as

$$
\tilde{x}_{i}(t \mid t-1) \triangleq x(t)-\hat{x}_{i}(t \mid t-1)
$$

Provided the $i$ th group of sensors is healthy (i.e., $\Pi_{i}=I_{p_{i}}$ ), the associated prediction error (20) satisfies, using (1), (4), (7) and (8)

$\tilde{x}_{i}(t+1 \mid t)=\left(A-A M_{i} C_{i}\right) \tilde{x}_{i}(t \mid t-1)+\left[\begin{array}{ll}E & -A M_{i}\end{array}\right]\left[\begin{array}{c}w(t) \\ \eta_{i}(t)\end{array}\right]$

Note that, due to observability of the pair $\left(A, C_{i}\right)$, for $i=$ $1, \ldots, N$, the matrices

$$
A_{M_{i}} \triangleq A-A M_{i} C_{i}
$$

have their eigenvalues strictly inside the unit circle. Hence it follows that the estimation errors $\tilde{x}_{i}$ associated to healthy groups of sensors are bounded whenever $w$ and $\eta_{i}$ are bounded. Moreover, using a procedure in [5], [11], we can obtain invariant sets, denoted as $\Xi_{i}$, and ultimate bounds for the prediction errors of the form

$$
\left|\tilde{x}_{i}(t \mid t-1)\right| \leq \overline{\tilde{x}}_{i}
$$

where $\Xi_{i}$ and $\bar{x}_{i}$ are computed from equation (21) and the bounds (2) and (5) on the disturbance signals.

Note from (4) [with $\Pi_{i}=I_{p_{i}}$ ], (8), (13) and (20) that the estimation errors satisfy

$$
\tilde{x}_{i}(t \mid t)=\left(I_{n}-M_{i} C_{i}\right) \tilde{x}_{i}(t \mid t-1)-M_{i} \eta_{i}(t)
$$

Using the bounds (5) and (23), we can find ultimate bounds for $\tilde{x}_{i}(t \mid t)$ in (24) as:

$$
\left|\tilde{x}_{i}(t \mid t)\right| \leq \overline{\tilde{x}}_{i}^{\prime} \triangleq\left|I_{n}-M_{i} C_{i}\right| \overline{\tilde{x}}_{i}+\left|M_{i}\right| \bar{\eta}_{i}
$$

\section{E. Tracking errors}

We define the plant tracking error, $z(t)$, the prediction tracking errors, $\hat{z}_{i}(t \mid t-1)$, and the estimation tracking errors, $\hat{z}_{i}(t \mid t)$, for $i=1, \ldots, N$, as

$$
\begin{aligned}
z(t) & =x(t)-x_{r e f}(t) \\
\hat{z}_{i}(t \mid t-1) & =\hat{x}_{i}(t \mid t-1)-x_{r e f}(t) \\
\hat{z}_{i}(t \mid t) & =\hat{x}_{i}(t \mid t)-x_{r e f}(t)
\end{aligned}
$$

Note from (8) that prediction and estimation tracking errors are related as

$$
\hat{z}_{i}(t \mid t)=\hat{z}_{i}(t \mid t-1)+M_{i}\left[y_{i}(t)-C_{i} \hat{x}_{i}(t \mid t-1)\right]
$$

Also, using (18), the optimal fusion estimated tracking error is defined as

$$
\hat{z}_{f u s}(t) \triangleq \hat{x}_{f u s}(t)-x_{\text {ref }}(t)
$$

Using (13), (18), (19) and (26) the optimal fusion estimated tracking error (30) satisfies

$$
\begin{aligned}
\hat{z}_{f u s}(t) & =\sum_{k \in \mathbb{H}} \lambda_{k} \hat{x}_{k}(t \mid t)-\sum_{k \in \mathbb{H}} \lambda_{k} x_{r e f}(t) \\
& =\sum_{k \in \mathbb{H}} \lambda_{k}\left(z(t)-\tilde{x}_{k}(t \mid t)\right)=z(t)-\sum_{k \in \mathbb{H}} \lambda_{k} \tilde{x}_{k}(t \mid t)
\end{aligned}
$$

The variable $\hat{z}_{\text {fus }}(t)$ will be applied in a state feedback tracking controller, as explained in Section II-F below.

\section{F. Feedback tracking control}

To achieve the tracking objective described in Section II-A we will employ a state feedback controller which computes the following control action using the optimal fused estimates

$$
\begin{aligned}
u(t) & =-K \hat{z}_{f u s}(t)+u_{r e f}(t) \\
& =-K\left(\hat{x}_{f u s}(t)-x_{r e f}(t)\right)+u_{r e f}(t)
\end{aligned}
$$

where the gain $K$ is obtained using any state feedback design technique (e.g., pole placement, $\mathrm{LQR}$ ) on the system $(A, B)$.

Assumption 2.3: The matrix

$$
A_{K} \triangleq A-B K
$$

has all its eigenvalues strictly inside the unit circle. (For this to be possible we will assume that the pair $(A, B)$ is stabilisable.)

\section{Fault Detection and Isolation}

In this section we describe the proposed fault detection and isolation principle. The principle is based on the separation of "healthy" sets, where the estimation tracking errors (28) remain under healthy operation, from "under-fault" sets, towards which the estimation tracking errors jump when abrupt sensor faults occur in one or more groups of sensors. The computation of these sets, as well as the derivation of conditions to achieve the aforementioned separation, requires the analysis of the closed-loop system dynamics under the proposed fusion-estimate-based feedback controller both for healthy and under-fault operation. In this analysis, performed in the following subsections, we will assume that the FDI 
correctly identifies the faulty groups of sensors, so that the fusion estimate (18) is only formed by estimations corresponding to healthy groups of sensors. Later, in Theorem 3.5, we will provide conditions that guarantee the correct selection of healthy groups of sensors by the FDI, thus validating the analysis.

In contrast with other schemes, (see, e.g., [9], [10]), which use stochastic arguments for fault detection and control reconfiguration, the approach followed here is very simple computationally since, once the required conditions are satisfied by design (off-line), the on-line system complexity only depends on the number of different fault situations considered.

\section{A. Closed-loop stability under healthy sensor fusion}

We first establish closed-loop stability of the multisensor fusion scheme described in Section II when only healthy sensors are used to compute the fused estimate (18). By using (1), (3), (26), (31)-(33) we obtain

$$
z(t+1)=A_{K} z(t)+B K \sum_{k \in \mathbb{H}} \lambda_{k} \tilde{x}_{k}(t \mid t)+E w(t)
$$

Therefore, since $\tilde{x}_{i}(t \mid t)$ (for healthy groups of sensors) and $w(t)$ are bounded signals, it follows from Assumption 2.3 that the states of the system (34) are also bounded. Moreover, using the procedure in [5], [11], we can obtain an ultimate bound for the tracking error $z(t)$ of the form

$$
|z(t)| \leq \bar{z}
$$

where $\bar{z}$ is computed from the dynamic equation (34) and the bounds ${ }^{2}(2)$ and (25) on its input signals.

\section{B. Prediction and estimation tracking errors associated with healthy sensors}

We develop the dynamics of the prediction tracking errors $\hat{z}_{i}(t \mid t-1)$ (see (27)), for $i \in \mathbb{H}$, in order to obtain attractive invariant sets and ultimate bounds for these variables and for the estimation tracking errors $\hat{z}_{i}(t \mid t)$ (see (28)) when only healthy sensors are used to compute the fused estimate (18). By using (3), (4) (with $\Pi_{i}=I_{p_{i}}$ ), (7), (8), (26), (27), (31) and (32) we obtain

$\hat{z}_{i}(t+1 \mid t)=A_{M_{i}} \hat{z}_{i}(t \mid t-1)+B_{1} z(t)+B_{2}\left[\begin{array}{c}\sum_{k \in \mathbb{H}} \lambda_{k} \tilde{x}_{k}(t \mid t) \\ \\ \eta_{i}(t)\end{array}\right]$

where $A_{M_{i}}$ is as defined in (22) and $B_{1}=A M_{i} C_{i}-B K$, $B_{2}=\left[\begin{array}{ll}B K & A M_{i}\end{array}\right]$. Note that the inputs to (36), namely,

$$
w_{i}(t) \triangleq\left[\begin{array}{lll}
z^{T}(t) & \sum_{k \in \mathbb{H}}\left(\lambda_{k} \tilde{x}_{k}(t \mid t)\right)^{T} & \eta_{i}^{T}(t)
\end{array}\right]^{T}
$$

can be bounded componentwise as $\left|w_{i}(t)\right| \leq \bar{w}_{i}$ using the bounds (35), (25) and (5). Then, since the matrices $A_{M_{i}}$ have all their eigenvalues inside the unit circle, the prediction tracking error $\hat{z}_{i}(t \mid t-1)$ is also bounded. Using a procedure in [5], we can compute the following attractive invariant set

\footnotetext{
${ }^{2}$ Note, in particular, that the bound (25) applies, since we have assumed that only healthy sensors take part in the fusion estimate.
}

for each healthy sensors-estimator combination in which, in the absence of sensor faults, the trajectories of (36) will remain if started inside or towards which they will converge if started outside:

$$
\Omega_{i}=\left\{\hat{z}_{i} \in \mathbb{R}^{n}:\left|V_{i}^{-1} \hat{z}_{i}\right| \leq r_{i}+\epsilon_{i}\right\}
$$

where $A_{M_{i}}=V_{i} \Lambda_{i} V_{i}^{-1}$ is the Jordan decomposition of $A_{M_{i}}$, $r_{i}=\left(I_{p_{i}}-\left|\Lambda_{i}\right|\right)^{-1}\left|V_{i}^{-1}\left[\begin{array}{ll}B_{1} & B_{2}\end{array}\right]\right| \bar{w}_{i}$ and $\epsilon_{i} \in \mathbb{R}^{n}$ is any (arbitrarily small) vector with positive components. (38) can be refined by means of the contractive procedure of [11].

Next, substituting (4) (with $\Pi_{i}=I_{p_{i}}$ ) in (29), and using (20), we have that the estimation tracking errors (28), for $i \in \mathbb{H}$, satisfy

$$
\hat{z}_{i}(t \mid t)=\hat{z}_{i}(t \mid t-1)+M_{i} C_{i} \tilde{x}_{i}(t \mid t-1)+M_{i} \eta_{i}(t)
$$

Thus, $\hat{z}_{i}(t \mid t)$, for $i \in \mathbb{H}$, belong to the sets ${ }^{3}$

$$
\Gamma_{i} \triangleq \Omega_{i} \oplus M_{i} C_{i} \Xi_{i} \oplus M_{i} \mathcal{N}_{i}
$$

where $\Xi_{i}$ is defined in Section II-D and $\mathcal{N}_{i}$ is a bounding box associated with the noise bounds (5), whenever the prediction errors (20) belong to the invariant sets $\Xi_{i}$ defined in Section II-D and the prediction tracking errors (27) belong to the invariant set (38).

\section{Conditions for fault tolerance}

Consider a fault in the $j$ th group of sensors, characterised by a change of the fault matrix $\Pi_{j}$ in (4) from the identity matrix (healthy case, see (6)) to a new "under fault" value. At the time of the fault, substituting (4) into (29), and using (20) and (27), we have that the "under fault" estimation tracking error $\hat{z}_{j}^{F}(t \mid t)$ satisfies

$$
\begin{aligned}
& \hat{z}_{j}^{F}(t \mid t)=\left[I_{n}+M_{j}\left(\Pi_{j}-I_{p_{j}}\right) C_{j}\right] \hat{z}_{j}(t \mid t-1) \\
& +M_{j}\left(\Pi_{j}-I_{p_{j}}\right) C_{j} x_{r e f}(t)+M_{j} \Pi_{j} C_{j} \tilde{x}_{j}(t \mid t-1) \\
& \quad+M_{j}\left[\Pi_{j} \eta_{j}(t)+\left(I_{p_{j}}-\Pi_{j}\right) \eta_{j}^{F}(t)\right]
\end{aligned}
$$

Thus, provided the prediction tracking error $\hat{z}_{j}(t \mid t-1)$ defined in (27) belongs to the invariant set (38) and the prediction error (20) belongs to the invariant set $\Xi_{i}$, then the estimation tracking error $\hat{z}_{j}^{F}(t \mid t)$ at the time of the fault belongs to the following set

$$
\begin{aligned}
\Gamma_{j}^{F}\left(\Pi_{j}\right) \triangleq\left[I_{n}+M_{j}\left(\Pi_{j}-I_{p_{j}}\right) C_{j}\right] \Omega_{j} \\
\oplus M_{j}\left(\Pi_{j}-I_{p_{j}}\right) C_{j} X_{r e f} \oplus M_{j} \Pi_{j} C_{j} \Xi_{j} \\
\quad \oplus M_{j} \Pi_{j} \mathcal{N}_{j} \oplus M_{j}\left(I_{p_{j}}-\Pi_{j}\right) \mathcal{N}_{j}^{F}
\end{aligned}
$$

where $X_{\text {ref }}$ is as in Assumption 2.1 and $\mathcal{N}_{j}$ and $\mathcal{N}_{j}^{F}$ are bounding boxes associated with the noise bounds (5). Note that expressions (41)-(42) coincide with (39)-(40) in the healthy case (when $\Pi_{j}=I_{p_{j}}$ ).

To ensure that the sets $\Gamma_{i}$ and $\Gamma_{i}^{F}\left(\Pi_{i}\right)$ characterise the estimation tracking errors under healthy operation and at the time of the fault, respectively, we assume the following.

Assumption 3.1: Before the occurrence of any sensor fault, the system has been operating under healthy condition

\footnotetext{
${ }^{3}$ The symbol $\oplus$ denotes the Minkowski sum of sets.
} 
for a sufficiently long time such that all the prediction error trajectories are inside the attractive invariant sets $\Xi_{i}$ defined in Section II-D and all prediction tracking error trajectories are inside the attractive invariant sets $\Omega_{i}$ defined in (38), for $i=1, \ldots, N$. Moreover, we assume that at least one sensor is operational at all time.

In order to ensure an effective fault detection and isolation by the FDI criterion, given later, we need to verify that the sets $\Gamma_{j}$ and $\Gamma_{j}^{F}\left(\Pi_{j}\right)$ are separated.

Assumption 3.2: The condition $\Gamma_{j} \cap \Gamma_{j}^{F}\left(\Pi_{j}\right)=\emptyset$ holds for all $j=1, \ldots, N$, for any of the possible values ${ }^{4}$ of the fault matrix $\Pi_{j}$ characterising the considered fault situation for the $j$ th group of sensors.

Note that the sets $\Gamma_{i}$ given by (40) are centred at 0 (this is so because the sets $\Omega_{i}$ given in (38); $\Xi_{i}$ defined by the dynamics (21); and $\mathcal{N}_{i}$ defined as a bounding box associated with the noise bounds (5), are all centred at 0 ). The set $\Gamma_{j}^{F}\left(\Pi_{j}\right)$, on the other hand, is offset around a centre point $c_{j}\left(\Pi_{j}\right)$ given by

$$
c_{j}\left(\Pi_{j}\right)=M_{j}\left(\Pi_{j}-I_{p_{j}}\right) C_{j} x_{r e f, 0}
$$

where $x_{r e f, 0}$ is as in Assumption 2.1. Thus, the reference offset $x_{r e f, 0}$ - which in turn shifts the centre $c_{j}\left(\Pi_{j}\right)$ in (43)provides a mechanism to achieve the set separation condition of Assumption 3.2.

Remark 3.3: Notice that Assumption 3.1 guarantees that when the fault in the $j$ th sensor group occurs at some time instant $t, \tilde{x}_{j}(t \mid t-1)$ is in $\Xi_{j}$ and $\hat{z}_{j}(t \mid t-1)$ is in $\Omega_{j}$ (the invariant sets corresponding to the healthy operation of the $j$ th sensor). Hence we have from (42) that, at the time of the fault, $\hat{z}_{j}^{F}(t \mid t) \in \Gamma_{j}^{F}\left(\Pi_{j}\right)$. Combining this condition with Assumption 3.2, we conclude that the $i$ th group of sensors, for $i \in\{1, \ldots, N\}$, is healthy at any time $t$ (and thus can be fused) if $\hat{z}_{i}(t \mid t) \in \Gamma_{i}$; and that the moment $\hat{z}_{i}(t \mid t)$ leaves the set $\Gamma_{i}$ allows us to detect a fault in that sensor group which, in consequence, must be discarded.

Based on the above developments, the fault diagnosis criterion proposed for the FDI unit is as follows:

Criterion 3.4 (FDI): At each time step, for each $i=$ $1, \ldots, N$ : if the estimation tracking error satisfies $\hat{z}_{i}(t \mid t) \in$ $\Gamma_{i}$ then the $i$ th group of sensors is deemed healthy and considered for fusion in (18); if $\hat{z}_{i}(t \mid t) \notin \Gamma_{i}$ then the $i$ th group of sensors is deemed faulty and discarded for all future times.

We then have the following result.

Theorem 3.5: Consider the multisensor fusion control scheme described in Section II. Under the conditions stated in Assumptions 2.3, 3.2 and 3.1, the system (1) with control (32) reconfigured by the use of the FDI Criterion 3.4 to select the index set $\mathbb{H}$ in (17) [used to compute the optimal fused estimates (18)], preserves closed-loop stability whenever a $j$ th group of sensors fails with fault matrix $\Pi_{j}$. Moreover, in the absence of disturbances and measurement noise in the healthy sensors, the tracking error $z(t)$ converges asymptotically to zero.

\footnotetext{
${ }^{4}$ Note that, depending on the problem characteristics, more than one value of the fault matrix $\Pi_{j}$ can be considered for the $j$ th group of sensors.
}

Proof: (Outline) As explained in Remark 3.3, Assumptions 3.2 and 3.1 guarantee that the FDI Criterion 3.4 only selects healthy groups of sensors to compute the optimal fused estimates (18) used in the control law (32). Thus, the analysis of Sections III-A to III-C is validated, achieving the desired boundedness and stability requirements.

Moreover, in the absence of disturbances, $w(t) \equiv 0$, and of measurement noise in healthy sensors, $\eta_{i}(t) \equiv 0$ for $i \in \mathbb{H}$, we conclude from (21) and the stability of the matrix $A_{M_{i}}$, that the prediction errors $\tilde{x}_{i}(t \mid t-1)$ for $i \in \mathbb{H}$ converge asymptotically to zero. Equation (24) then implies that the estimation errors $\tilde{x}_{i}(t \mid t)$ also converge asymptotically to zero. Then, we have from (34) and Assumption 2.3 that $z(t)$ converges asymptotically to zero.

\section{EXAMPLE}

Consider a system of the form (1), (4), with matrices:

$$
\begin{aligned}
& A=\left[\begin{array}{ccc}
1.1269 & -0.4940 & 0.1129 \\
1 & 0 & 0 \\
0 & 1 & 0
\end{array}\right] ; B=\left[\begin{array}{c}
-0.3832 \\
0.5919 \\
0.5191
\end{array}\right] \\
& E=\left[\begin{array}{c}
-0.3832 \\
0.5919 \\
0.5191
\end{array}\right] ; C_{1}^{T}=\left[\begin{array}{l}
1 \\
0 \\
0
\end{array}\right] ; C_{2}^{T}=\left[\begin{array}{l}
0 \\
1 \\
0
\end{array}\right] ; C_{3}^{T}=\left[\begin{array}{l}
0 \\
0 \\
1
\end{array}\right]
\end{aligned}
$$

For the design of the Kalman filter state estimator (7)-(11), the process disturbance and measurement noises are assumed Gaussian white noises, independent from each other, and with zero mean and variances $Q=1.0322 \times 10^{-5}, R_{1}=$ $4.3331 \times 10^{-6}, R_{2}=R_{3}=8.6662 \times 10^{-6}$. In this example, the bounds on the process disturbances and measurement noises are taken as:

$|w(t)| \leq 1 e^{-2},\left|\eta_{i}(t)\right| \leq 1 e^{-2},\left|\eta_{i}^{F}(t)\right| \leq 1 e^{-2}, i=1,2,3$,

In order to reconcile the above two assumptions, all stochastic sequences have been generated using a random generator with zero-mean Gaussian distribution. Then, the entire sequences were scaled in order to satisfy the respective boundedness assumptions and so that the variances coincided with the values given above.

The objective is for the first component of the state, $x_{1}(t)$, to track a square-like wave, which results in a reference for the state vector, $x_{r e f}(t)$, satisfying the following bounds:

$$
\left[\begin{array}{lll}
19 & 13.8038 & 9.4268
\end{array}\right]^{T} \leq x_{r e f} \leq\left[\begin{array}{lll}
21 & 15.25 & 10.22
\end{array}\right]^{T}
$$

The steady-state Kalman estimators parameters $\left(M_{i}, P_{i i}\right.$, $\left.P_{i j}\right)$ are designed as in Section II . For the fusion estimator, when all three sensors are deemed healthy by the FDI (corresponding to an FDI decision index equal to 0 ) the error crosscovariance is computed using all the local sensor-estimator subsystem covariances. When the $j$ th sensor is deemed faulty by the FDI, $j \in\{1,2,3\}$ (corresponding to an FDI decision index equal to $j$ ) the error cross-covariance is determined by using the remaining sensor-estimator subsystem covariances. The feedback gain $K=\left[\begin{array}{lll}0.0358 & 0.1173 & -0.0979\end{array}\right]$ is computed as described in Section II-F using pole placement. 
In the sequel we illustrate the performance of the proposed fault tolerant multisensor fusion control scheme applied to the above system. The following fault scenario is considered:

1) All the sensors start under healthy operation.

2) We assume an outage of Sensor 1 at time $t=50 \mathrm{~s}$, so that the fault parameter in (4) is $\Pi_{1}=0$. Then, the sensor recovers ${ }^{5}$ at time $t=150 \mathrm{~s}$.

3) We assume an outage of Sensor 2 at time $t=200 \mathrm{~s}$, so that the fault parameter in (4) is $\Pi_{2}=0$. Then, the sensor recovers at time $t=300 \mathrm{~s}$.

4) We assume an outage of Sensor 3 at time $t=350 \mathrm{~s}$ and remains in this state, so that the fault parameter in (4) is $\Pi_{3}=0$.

Fig. 1 shows the sets $\Gamma_{1}$ defined in (40) (green sets centred at zero) and $\Gamma_{1}^{F}\left(\Pi_{1}\right)$ defined in (42) (red sets centred away from zero). Notice that the set separation condition of Assumption 3.2 is satisfied for Sensor 1. A similar test is carried out for Sensors 2 and 3, and the condition stated in (3.2) still holds. Thus, when a fault occurs in the $j$ th sensor (for $j=1,2,3)$ as described in the list above, the corresponding estimation tracking error trajectories jump from the set $\Gamma_{j}$ to the "shifted" set $\Gamma_{j}^{F}\left(\Pi_{j}\right)$, making fault diagnosis possible. According to Theorem 3.5, we can conclude that the scheme preserves closed-loop stability whenever a sensor fails under the above considered fault situation.

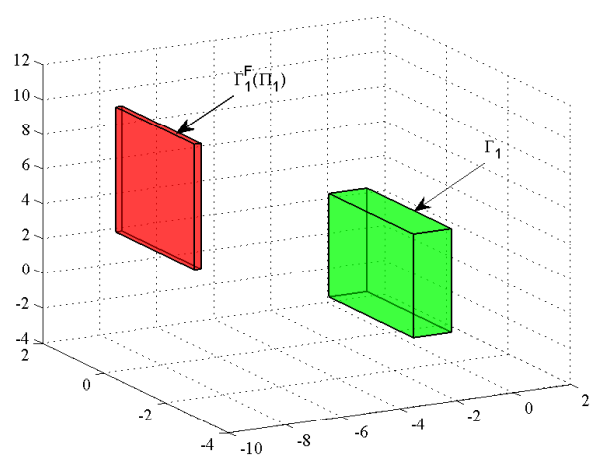

Fig. 1. Separation of sets representing healthy and faulty behaviour.

Fig. 2 shows the simulation results. Subplot (a) shows the FDI decision index. Fig. 2 (b) shows the effectiveness of the scheme as the reference signal (dash-dotted red line) is accurately tracked by the first component of the system state (dash blue line) under all fault situations considered. Subplots (c), (d), (e) show the estimation tracking errors $\hat{z}_{1}(t \mid t), \hat{z}_{2}(t \mid t)$, $\hat{z}_{3}(t \mid t)$ using measurements from, respectively, sensors 1,2 , 3 . Note that all three estimator tracking errors are shifted from 0 after the occurrence of a fault.

\section{CONCLUSION}

In this paper, a multisensor fusion fault tolerant control strategy based on set separation to achieve fault detection

\footnotetext{
${ }^{5}$ Although we have not considered the possibility of recovery of a sensor (that is, its reversion to healthy operation), the simulation results illustrate that the scheme is capable of handling more challenging fault scenarios than the one considered in the theoretical analysis.
}

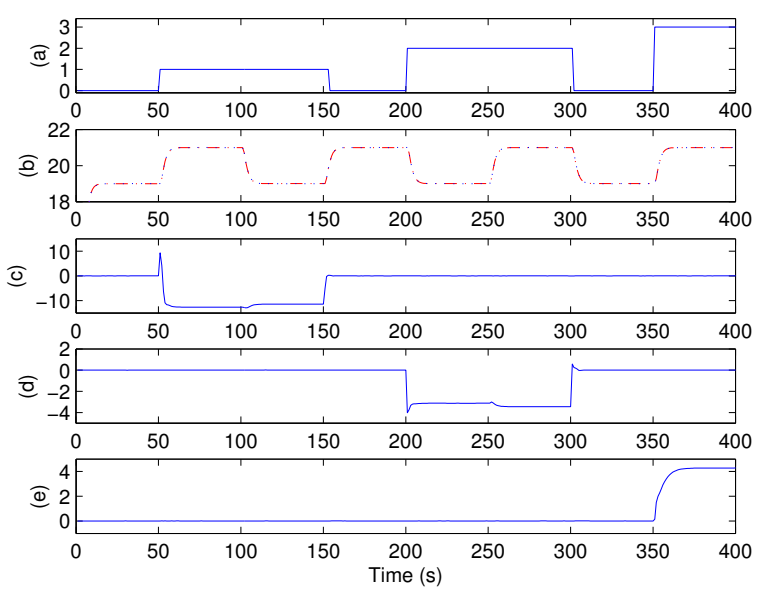

Fig. 2. Performance of the fault tolerant multisensor fusion scheme under the considered fault scenario.

and isolation (FDI) is proposed. The FDI module provides a mechanism where the estimation tracking errors of each sensor is tested for containment in a pre-computed "healthy" set. If the trajectories for a sensor estimation tracking error belong to a pre-computed "under-fault" set which is separated from the "healthy" one, the sensor is deemed to be faulty and is discarded from the closed-loop feedback control. Only healthy sensor estimates are fused in a twolayer fusion scheme in order to obtain the optimal fusion estimate used by the controller. We have proven closed-loop stability and the fault tolerance capabilities of the scheme.

\section{REFERENCES}

[1] B.V. Dasarathy. Sensor fusion potential exploitation-Innovative architectures and illustrative applications, Proceedings of the IEEE, 85(1):24-38, January 1997.

[2] S.L Sun and Z.L. Deng, Multisensor optimal information fusion Kalman filter, Automatica, 2004, 40, pp.1017-1023.

[3] Lijun Xu, Jian Qiu Zhang, and Yong Yan. A wavelet-based multisensor data fusion algorithm. IEEE Transactions on Instrumentation and Measurement, 53(6):1539-1545, 2004

[4] M. Blanke, M. Kinnaert, J. Lunze, and M. Staroswiecki. Diagnosis and Fault Tolerant Control. Springer-Verlag Berlin Heidelberg, 2003. ISBN 3-540-01056-4.

[5] E. Kofman, H. Haimovich, and M. M. Seron. A systematic method to obtain ultimate bounds for perturbed systems. International Journal of Control, 80(2):167-178, 2007.

[6] S.L Sun and Z.L. Deng, Distributed optimal fusion steady-state Kalman filter for systems with coloured measurement noises, International Journal of Systems Science, Vol. 36, No. 3, 20 February 2005, $113-118$

[7] Zhu, Y., You, Z. H., Zhao, J., Zhang, K. S., and Li, X. R. The optimality for the distributed Kalman filtering fusion with feedback. Automatica, 2001, 37, 1489-1493

[8] Saha, R. K., and Chang, K. C. Efficient algorithm for multisensor track fusion. IEEE Transactions on Aerospace and Electronic Systems, 34(1), 200-210, 1998.

[9] C. Hajiyev and F. Caliskan. Sensor/actuator fault diagnosis based on statistical analysis of innovation sequence and robust Kalman filtering.Aerosp. Sci. Technol., 4:415-422, 2000.

[10] E.C. Larson, B.E. Parker Jr, and B.R. Clark. Model-based sensor and actuator fault detection and isolation. In Proceedings of the American Control Conference, Anchorage, AK, May 2002.

[11] S. Olaru, J.A. De Doná, and M.M. Seron. Positive invariant sets for fault tolerant multisensor control schemes. Proceedings 17 IFAC World Congress, Seoul, Korea, July 2008. 